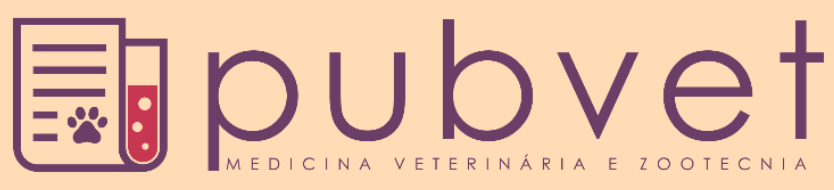

https://doi.org/10.31533/pubvet.v15n03a776.1-5

\title{
Alterações hematológicas em cães positivos para Leishmania spp em esfregaço sanguíneo
}

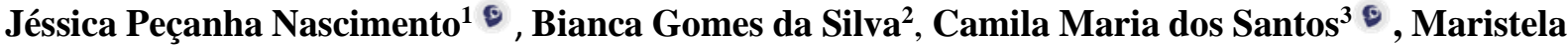 \\ Martins Halverson ${ }^{4}$ ๑ \\ ${ }^{1}$ Aprimoranda em Patologia Clínica na Clínica Veterinária da Universidade Anhanguera Uniderp - Campo Grande, MS. \\ ${ }^{2}$ Aprimoranda em Clínica Médica e Cirúrgica, na Clínica Veterinária da Universidade Anhanguera Uniderp - Campo Grande, MS. \\ ${ }^{3}$ Mestranda do Programa de Pós Graduação em Doenças Infecciosas e Parasitárias da Faculdade de Medicina da \\ Universidade Federal de Mato Grosso do Sul (UFMS) - Campo Grande, MS \\ ${ }^{4}$ Mestre em Ciências Veterinárias pela UFMS. Docente da Universidade Anhanguera Uniderp - Campo Grande, MS.
}

Resumo. O presente estudo analisou as principais alterações hematológicas de cães positivos para Leishmania spp em esfregaço sanguíneo atendidos na clínica veterinária da UNIDERP no período de fevereiro de 2010 a outubro de 2019. Foram atendidos 55 (100\%) cães positivos para Leishmania em esfregaço sanguíneo, sendo que as principais alterações encontradas foram a presença de anemia, hiperproteinemia, linfopenia, trombocitopenia, neutrofilia, eosinopenia, presença de rouleaux eritrocitário e leucocitose. As alterações hematológicas tornam-se importantes para as áreas endêmicas para possível suspeita de Leishmaniose.

Palavras chave: Leishmaniose, hematologia, epidemiologia

\section{Hematological changes in dogs positive for Leishmania spp in a blood smear}

Abstract. The present study analysed hematological alterations present in fifty-five canine subjects found to be Leishmania positive based on blood smear analysis at the UNIDERP veterinary clinic in Campo Grande, MS Brazil, between February 2010 and October 2019. The principal alterations detected included; anemia, hyperproteinemia, lymphopenia, thrombocytopenia, neutrophilia, eosinopenia, presence of erythrocyte rouleaux and leukocytosis. The presence of these hematological conditions can assist health professionals in the identification of Leishmania in areas where the disease is endemic.

Keywords: Leishmaniasis, hematology, epidemiology

\section{Alteraciones hematológicas en perros positivos para Leishmania spp en un frotis de sanguíneo}

Resumen. El presente estudio analizó los principales cambios hematológicos de perros positivos a Leishmania spp en un frotis de sangre atendidos en la clínica veterinaria UNIDERP desde febrero de 2010 a octubre de 2019. Se observaron 55 (100\%) perros positivos a Leishmania en un frotis de sangre, que los principales cambios encontrados fueron la presencia de anemia, hiperproteinemia, linfopenia, trombocitopenia, neutrofilia, eosinopenia, presencia de eritrocitos rouleaux y leucocitosis. Los cambios hematológicos se vuelven importantes para las áreas endémicas por una posible sospecha de leishmaniasis.

Palabras clave: Leishmaniasis, hematología, epidemiología 


\section{Introdução}

A leishmaniose é uma doença infecciosa não contagiosa causada pelo parasita do gênero Leishmania spp. Está inserido no grupo dos protozoários e pertence à família Trypanosomatidae. Infecta células do sistema imune, principalmente monócitos, onde ocorre a reprodução e disseminação do parasita. Suas duas formas de vida são promastigota (encontrada no vetor) e amastigota (encontrada nos tecidos do hospedeiro vertebrado) (Brasil, 2017).

Considerada cosmopolita, a leishmaniose já foi descrita em diferentes localidades, um trabalho de revisão de 2012 (Alvar et al., 2012), demonstra a presença do agente em 98 países. A falta de dados de notificação de incidência prejudica a análise epidemiológica, países só são considerados endêmicos quando uma espécie sabidamente patogênica ao ser humano é descrita sendo que infecções humanas com baixa carga parasitária não são consideradas na notificação, isso gera dados incertos sobre a verdadeira perspectiva da enfermidade (Alvar et al., 2012).

A Leishmania consegue se reproduzir e se disseminar muito facilmente no cão, por isso, este é considerado o principal reservatório, entretanto, os felinos domésticos já estão sendo investigados como potenciais reservatórios do parasita (Rocha et al., 2019), e desde 2004 existem trabalhos demonstrando a leishmaniose como doença nessa espécie (Schubach et al., 2004). Estudos em diferentes regiões do país relatam a positividade em outros mamíferos, como por exemplo o trabalho realizado na cidade de Uruguaiana (RS) onde onze equinos foram confirmados através de análise molecular (Escobar et al., 2020). A forma mais conhecida de transmissão é através do flebotomíneo, sendo a espécie mais conhecida o Lutzomyia longipalpis. Outras 90 espécies de flebotomíneos já foram descritas como capazes de transmitir o parasita. O protozoário fica na saliva do vetor e pela picada é transmitido para o hospedeiro vertebrado, em períodos crepusculares e por fêmeas do feblotomíneo. Mais de setenta espécies já foram relatadas como hospedeiros definitivos (OIE, 2014).

É uma doença de caráter zoonótico, tendo seu agravo como consequência o óbito do paciente (Donato et al., 2013). De acordo com os dados do SINAN (Sistema de Informação de Agravos de Notificação) de 2001 a 2014 foram registrados 47.859 novos casos de leishmaniose visceral no Brasil, representando uma incidência de 1,8 por 100.00 habitantes (Reis et al., 2017).

Estados considerados livres da doença já estão relatando a presença do agente. Em Porto Alegre (RS) seis casos em humanos foram notificados, de 2016 a 2017, todos autóctones, desses, quatro vieram a óbito pouco tempo após a confirmação do diagnóstico por punção aspirativa de medula óssea. A explicação para o surgimento da doença se baseia na divisa com a Argentina, o trânsito de animais vindos do país vizinho pode ter facilitado a disseminação (Mahmud et al., 2019).

Alterações laboratoriais como anemia normocítica normocrômica arregenerativa, trombocitopenia ou alterações no leucograma podem indicar a suspeita de leishmaniose em cães, assim como alterações urinárias e lesões em diferentes partes do corpo (Paltrinieri et al., 2016). Mas, sempre se faz necessário o uso de métodos de diagnóstico para confirmação. Alguns testes apresentam melhores resultados em animais com apresentação de sintomatologia clínica, o exame parasitológico ainda demonstra ser um teste de escolha quando comparado a outras categorias (Pessoa-e-Silva et al., 2019). Métodos moleculares estão sendo cada vez mais empregados, em sua maioria a nível de pesquisa, podendo ser o diagnóstico de escolha devido à dificuldade em relação aos métodos sorológicos (Galluzzi et al., 2018).

Uma forma efetiva de controle e prevenção da infecção por leishmaniose que está sendo discutida recentemente é o uso de coleiras impregnadas por deltametrina. Já foi comprovada a queda na incidência de casos humanos e de animais com o uso contínuo e controlado da ferramenta, mais trabalhos em breve serão divulgados pelo Brasil (Coura-Vital et al., 2018).

Com a falta de informações sobre os aspectos hematológicos e bioquímicos de cães infectados naturalmente por leishmaniose, este trabalho tem como objetivo descrever as características laboratoriais de cães diagnosticados através de esfregaço sanguíneo na Clínica Veterinária da Universidade Anhanguera Uniderp, no período de fevereiro de 2010 a outubro de 2019. 


\section{Material e métodos}

As amostras de sangue foram obtidas a partir de punção da veia jugular e cefálica, coletados em tubos contendo ácido etilenodiaminotretrácetico (EDTA) e enviadas ao laboratório, onde foi realizado hemograma completo bem como esfregaço sanguíneo, fixado e corado por panótico para contagem diferencial de leucócitos, análise morfológica celular e pesquisa de hemoparasitos.

Os valores de eritrócitos e leucócitos totais foram obtidos manualmente pela câmera de Neubauer (hemocitômetro) e os valores de plaquetas, obtidos a partir do esfregaço sanguíneo, contagem de 10 campos em objetiva de (100x), valor dividido por 10, e multiplicado por 20.000.

O valor do hematócrito foi obtido através de micro centrifugação por meio de capilar sanguíneo, já o valor de hemoglobina através da cianometahemoglobina com leitura por analisador bioquímico (BIOPLUS, modelo BIO-200 ${ }^{\circledR}$ ).

A contagem diferencial de leucócitos foi realizada sob microscopia óptica, em objetiva de imersão (100x).

\section{Resultados e discussão}

Foram encontrados $55(100 \%)$ cães positivos com presença de amastigotas de Leishmania spp em esfregaço sanguíneo. Sendo cães de diversas raças, sexo e idade, atendidos na Clínica Veterinária UNIDERP entre fevereiro de 2010 a outubro de 2019. A tabela 1 monstra as principais alterações hematológicas encontradas tanto no eritrograma quanto no leucograma onde destacam-se anemia, hiperproteinemia, trombocitopenia, leucocitose, neutrofília, linfopenia e eosinopenia.

Tabela 1: Alterações hematológicas encontradas no hemograma de cães de diversas raças, sexo e idade, atendidos na Clínica Veterinária UNIDERP entre fevereiro de 2010 a outubro de 2019.

\begin{tabular}{|c|c|c|}
\hline Alterações em eritrograma & $\mathrm{N}^{\mathrm{o}}$ de animais & $\%$ do total \\
\hline Anemia & 47 & $85,4 \%$ \\
\hline Trombocitopenia & 39 & $70,9 \%$ \\
\hline Trombocitose & 2 & $3,6 \%$ \\
\hline Hiperproteinemia & 26 & $47,3 \%$ \\
\hline Hipoproteinemia & 8 & $14,5 \%$ \\
\hline Macroplaquetas & 17 & $30,9 \%$ \\
\hline Ictéricia & 10 & $18,2 \%$ \\
\hline Alterações em leucograma & $\mathrm{N}^{\mathrm{o}}$ de animais & $\%$ do total \\
\hline Leucocitose & 16 & $20,1 \%$ \\
\hline Leucopenia & 9 & $16,4 \%$ \\
\hline Desvio nuclear de neutrófilos a esquerda & 17 & $30,9 \%$ \\
\hline Neutrofília & 21 & $38,2 \%$ \\
\hline Neutropenia & 3 & $5,5 \%$ \\
\hline Linfopenia & 35 & $63,6 \%$ \\
\hline Eosinofília & 1 & $1,8 \%$ \\
\hline Eosinopenia & 29 & $52,7 \%$ \\
\hline Monocitose & 12 & $21,8 \%$ \\
\hline
\end{tabular}

A anemia é a diminuição da quantidade de eritrócitos circulantes, acarretando assim na diminuição de oxigenação tecidual (Thrall, 2015). A hiperproteinemia foi o segundo achado mais frequente seguido de trombocitopenia. Segundo Braz et al. (2015) de 153 cães avaliados, foi notado a presença de hiperproteinemia $(64,9 \%)$ e trombocitopenia $(50,0 \%)$.

Outro achado importante no eritrograma foi a presença de rouleaux eritrocitário, dos 55 (100\%) cães, $11(20 \%)$ apresentaram essa alteração. O rouleaux eritrocitário ocorre normalmente quando algumas proteínas do plasma estão aumentadas, como fibrinogênio e imunoglobulinas (Thrall, 2015).

A alteração mais frequente foi a linfopenia, presença de linfócitos reacionais (15 animais) e monocitose, de acordo com Medeiros et al. (2008), no estudo realizado também houve predominância de linfopenia, linfócitos reacionais e monocitose nas alterações leucocitárias mais frequentes.

No estudo realizado por Braz et al. (2015) a maioria dos animais apresentaram valores de leucócitos normais, já no presente trabalho a alteração leucocitária predominante foi a presença de leucocitose por neutrofília seguida de desvio a direita. 


\section{Conclusão}

A presença de anemia, hiper proteinemia e leucocitose com desvio a direita foram alterações que caracterizaram o padrão hematológico de cães positivos no esfregaço sanguíneo sendo alterações em hemograma de grande valia para áreas endêmicas quando se suspeitar de Leishmaniose.

\section{Agradecimentos}

Os autores agradecem o apoio da Uniderp e Funadesp.

\section{Referências}

Alvar, J., Vélez, I. D., Bern, C., Herrero, M., Desjeux, P., Cano, J., Jannin, J., \& den Boer, M. (2012). Leishmaniasis worldwide and global estimates of its incidence. PLOS ONE, 7(5), e35671. https://doi.org/10.1371/journal.pone.0035671.

BRASIL. Ministério da Saúde. Secretaria de Vigilância em Saúde. Departamento de Vigilância das Doenças Transmissíveis. Manual de vigilância da leishmaniose tegumentar [recurso eletrônico] / Ministério da Saúde, Secretaria de Vigilância em Saúde, Departamento de Vigilância das Doenças Transmissíveis. - Brasília: Ministério da Saúde, 2017. 189 p.: il. Edição eletrônica da $2^{\mathrm{a}}$ edição do livro: Manual de Vigilância da Leishmaniose Tegumentar Americana, atualizado.

Braz, P. H., Sartoretto, M. C., Souza, A. S., \& Melo, F. M. G. (2015). Perfil hematológico de cães naturalmente infectados por Leishmania spp. Acta Veterinaria Brasilica, 9(1), 87-90.

Coura-Vital, W., Leal, G. G. A., Marques, L. A., Pinheiro, A. D. C., Carneiro, M., \& Reis, A. B. (2018). Efetividade de coleiras impregnadas de deltametrina na incidência de infecção canina por Leishmania infantum: um estudo de intervenção em larga escala em uma área endêmica no Brasil. Plos One, 13(12). https://doi.org/10.1371/journal.pone.0208613.

Donato, L. E., Lima Júnior, F. E. F., Alburquerque, R., \& Gomes, M. L. S. (2013). Vigilância e controle de reservatórios da leishmaniose visceral no Brasil: aspectos técnicos e jurídicos. Revista de Educação Continuada Em Medicina Veterinária e Zootecnia Do CRMV-SP, 11(2), 18-23. https://doi.org/10.36440/recmvz.v11i2.16219.

Escobar, T. A., Dowich, G., Cantele, L. C., Zuravski, L., Ferrareze, P. A. G., Duarte, C. A., Kmetzsch Rosa e Silva, L., Lübeck, I., \& Manfredini, V. (2020). Molecular detection of Leishmania spp. in Brazilian cross-border south region mammalian hosts. Transboundary and Emerging Diseases, 67(2), 476-480. https://doi.org/10.1111/tbed.13361.

Galluzzi, L., Ceccarelli, M., Diotallevi, A., Menotta, M., \& Magnani, M. (2018). Real-time PCR applications for diagnosis of leishmaniasis. Parasites \& Vectors, 11(1), 1-13.

Mahmud, I. C., Piassini, L. de A. S., Motta, F., Behar, P. R. P., \& Souza, G. D. (2019). Epidemiological aspects of the first human autochthonous visceral leishmaniosis cases in Porto Alegre, Brazil. Brazilian Journal of Infectious Diseases, 23(2), 124-129. https://doi.org/10.1016/j.bjid.2019.04.004.

Medeiros, C. M. O., Melo, A. G. C., Lima, A. K. F., Silva, I. N. G., Oliveira, L. C., \& Silva, M. C. (2008). Perfil hematológico de cães com leishmaniose visceral nomunicípio de Fortaleza, Ceará. Ciência Animal, 18(1), 43-50.

OIE. (2014). World Organization for Animal Health (W. H. Organization (ed.)). World Health Organization.

Paltrinieri, S., Gradoni, L., Roura, X., Zatelli, A., \& Zini, E. (2016). Laboratory tests for diagnosing and monitoring canine leishmaniasis. Veterinary Clinical Pathology, 45(4), 552-578. https://doi.org/10.1111/vcp.12413.

Pessoa-e-Silva, R., Vaitkevicius-Antão, V., Andrade, T. A. S., Silva, A. C. O., Oliveira, G. A., TrajanoSilva, L. A. M., Nakasone, E. K. N., \& Paiva-Cavalcanti, M. (2019). The diagnosis of canine visceral leishmaniasis in Brazil: Confronting old problems. Experimental Parasitology, 199, 9-16. https://doi.org/10.1016/j.exppara.2019.02.012.

Reis, L. La, Balieiro, A. A. da S., Fonseca, F. R., \& Gonçalves, M. J. F. (2017). Changes in the epidemiology of visceral leishmaniasis in Brazil from 2001 to 2014. Revista Da Sociedade Brasileira de Medicina Tropical, 50(5), 638-645. 
Rocha, A. V. V. O., Moreno, B. F. S., Cabral, A. D., Louzeiro, N. M., Miranda, L. M., dos Santos, V. M. B., Costa, F. B., Nogueira, R. de M. S., Marcili, A., \& Sperança, M. A. (2019). Diagnosis and epidemiology of Leishmania infantum in domestic cats in an endemic area of the Amazon region, Brazil. Veterinary Parasitology, 273, 80-85.

Schubach, T. M. P., Figueiredo, F. B., Pereira, S. A., Madeira, M. F., Santos, I. B., Andrade, M. V, Cuzzi, T., Marzochi, M. C. A., \& Schubach, A. (2004). American cutaneous leishmaniasis in two cats from Rio de Janeiro, Brazil: first report of natural infection with Leishmania (Viannia) braziliensis. Transactions of the Royal Society of Tropical Medicine and Hygiene, 98(3), 165-167. https://doi.org/10.1016/S0035-9203(03)00040-3.

Thrall, M. A. (2015). Hematologia e Bioquímica Clínica Veterinária. In 2. ed. Editora Roca.

Histórico do artigo:

Recebido: 31 de agosto de 2020

Aprovado: 12 de outubro de 2020.

Disponível online: 25 de janeiro de 2021.
Licenciamento: Este artigo é publicado na modalidade Acesso Aberto sob a licença Creative Commons Atribuição 4.0 (CC-BY 4.0), a qual permite uso irrestrito, distribuição, reprodução em qualquer meio, desde que o autor e a fonte sejam devidamente creditados. 